\title{
Enzalutamide versus flutamide for castration-resistant prostate cancer after combined androgen blockade therapy with bicalutamide: study protocol for a multicenter randomized phase II trial (the OCUU-CRPC study)
}

Taro Iguchi', Satoshi Tamada, Minoru Kato, Sayaka Yasuda, Takeshi Yamasaki and Tatsuya Nakatani

\begin{abstract}
Background: Enzalutamide is an oral androgen receptor targeted agent that has been shown to improve survival in PREVAIL trials and has been approved for patients with chemo-naïve metastatic castration-resistant prostate cancer (CRPC). Meanwhile, flutamide is a non-steroidal oral anti-androgen that was commonly used before the approval of bicalutamide. The objective of the OCUU-CRPC study is to compare the efficacy and safety between second-line hormonal therapy of enzalutamide and flutamide as alternative anti-androgen therapy (AAT) after combined androgen blockade (CAB) therapy that included bicalutamide in patients with CRPC.

Methods: A total of 100 patients with CRPC with or without distant metastases after disease progression who received $C A B$ therapy with bicalutamide were randomly assigned at a 1:1 ratio according to distant metastases to the enzalutamide ( $160 \mathrm{mg} /$ day, $4 \times 40 \mathrm{mg}$ capsules once daily) and flutamide ( $375 \mathrm{mg} / \mathrm{day} ; 3 \times 125 \mathrm{mg}$ tablets thrice daily) groups. The primary endpoint for the drug efficacy is the response rate of prostate-specific antigen (PSA) (i.e., the ratio of patients whose PSA declined by $\geq 50 \%$ from baseline) at 3 months. Meanwhile, the secondary endpoints are PSA progression rate at 3 and 6 months, PSA response rate at 6 months, change in quality of life, PSA progression-free survival, and safety. The patient registration started in January 2015 and will end in March 2018, and the follow-up period is 6 months after the last patient registration. The main result will be reported in March 2019.

Discussion: In the OCUU-CRPC study, we compare the efficacy and safety of enzalutamide or alternative AAT with flutamide in participants with CRPC who were previously treated with a CAB therapy with bicalutamide. The expected results of this study will be that enzalutamide is superior to flutamide in terms of PSA response. A longer time to disease progression with enzalutamide over flutamide may translate to better overall survival. However, flutamide may be more accessible for patients owing to its lower cost than enzalutamide.
\end{abstract}

Trial registration: The OCUU-CRPC study was prospectively registered at clinicaltrials.gov (NCT02346578, January 2015) and University Hospital Medical Information Network (UMIN000016301, January 2015).

Keywords: Castration-resistant prostate cancer, Enzalutamide, Flutamide

\footnotetext{
* Correspondence: taro@msic.med.osaka-cu.ac.jp

Department of Urology, Osaka City University Graduate School of Medicine,

1-4-3 Asahi-machi, Abeno-ku, Osaka 545-8585, Japan
}

(c) The Author(s). 2019 Open Access This article is distributed under the terms of the Creative Commons Attribution 4.0 International License (http://creativecommons.org/licenses/by/4.0/), which permits unrestricted use, distribution, and reproduction in any medium, provided you give appropriate credit to the original author(s) and the source, provide a link to the Creative Commons license, and indicate if changes were made. The Creative Commons Public Domain Dedication waiver (http://creativecommons.org/publicdomain/zero/1.0/) applies to the data made available in this article, unless otherwise stated. 


\section{Background}

Prostate cancer is the most commonly diagnosed cancer and the third leading cause of cancer-related death among men worldwide [1]. In most patients who are treated for advanced recurrent prostate cancer with androgen-deprivation therapy (ADT) that comprise a luteinizing hormone-releasing hormone (LHRH) analogue or orchiectomy with or without an anti-androgen, disease progression occurs despite effective suppression of serum testosterone. These patients are then diagnosed with castration-resistant prostate cancer (CRPC).

In Japan, ADT has been widely used not only for advanced recurrent prostate cancer, but also for localized prostate cancer in elderly patients. Although ADT is effective only for a certain period and causes recurrence as CRPC, several patients actually respond to treatment for long periods [2]. Combined androgen blockade (CAB) therapy using an LHRH analogue with an anti-androgen is superior to ADT without an anti-androgen in terms of long-term efficacy among Japanese patients with prostate cancer [3]. Despite some disadvantages of CAB such as higher cost compared to ADT without anti-androgen, an observational study that compared $\mathrm{CAB}$ and castration showed good prognosis in patients with T1c-T3 prostate cancer [4]. At present, CAB using bicalutamide is more widely used in Japan than LHRH analogue monotherapy, and many elderly patients with localized prostate cancer are treated with CAB.

However, a proportion of patients with prostate cancer who are treated with $C A B$ experience prostate-specific antigen (PSA) recurrence and are diagnosed as CRPC after confirming for anti-androgen withdrawal syndrome (AWS). Alternative anti-androgen therapy (AAT) with flutamide as the subsequent therapy after $C A B$ therapy with bicalutamide is widely used before the androgen receptor targeted therapy (ART) era, particularly in Japan [5-11]. The response rate of AAT, defined as a decrease of $>50 \%$ from the baseline serum PSA level, was $22 \%$, and patients who respond to AAT have been reported to have good prognosis [6]. This phenomenon is attributed to the different mechanism of actions among anti-androgens [9].

Flutamide, a non-steroidal oral anti-androgen, was often used in clinical practice before bicalutamide was approved. Some small, single-arm non-randomized studies suggest a PSA benefit in flutamide as second-line hormonal therapy [5-11]. However, the use of flutamide is optional for limited patients with CRPC according to the American Urological Association guidelines [12] owing to the modest PSA benefit, with PSA declines of $>50 \%$ occurring typically in only $20-40 \%$ of men with a median duration measured in several months.

Enzalutamide is an androgen receptor inhibitor that targets several steps in the androgen receptor signaling pathway. It inhibits binding of androgens to the androgen receptor, androgen-receptor nuclear translocation, and androgen receptor-mediated DNA binding [13]. In preclinical studies, enzalutamide showed a higher affinity for the androgen receptor and superior suppression of key components of the androgen receptor signaling pathway than bicalutamide $[13,14]$. Subsequently, enzalutamide was approved for the treatment of metastatic CRPC based on the results of two pivotal placebo-controlled phase III trials, namely, AFFIRM [15] and PREVAIL [16].

Before the ART era, the treatment options for CRPC are limited, and AAT with flutamide has been widely used in Japan. However, no clinical studies compared AAT with flutamide and enzalutamide as treatment modalities for CRPC. Creating clinical evidence of the superiority of enzalutamide to AAT (bicalutamide to flutamide) in the post-AWS setting in terms of safety and efficacy would be meaningful. To the best of our knowledge, the OCUU-CRPC study is the first randomized head-to-head trial of enzalutamide versus flutamide in patients with CRPC after CAB therapy with bicalutamide.

\section{Methods/design}

\section{Aim, design, and setting of the study}

This study aims to compare the efficacy and safety of enzalutamide or AAT with flutamide in patients with CRPC who were previously treated with CAB therapy with bicalutamide. The efficacy and safety of enzalutamide and AAT with flutamide will be evaluated, and the effective therapy against CRPC after treatment with CAB therapy with bicalutamide will be investigated.

The present study is a phase II, investigator-initiated, multicenter, open-labeled randomized clinical trial of enzalutamide and AAT with flutamide in patients with CRPC after treatment with CAB therapy with bicalutamide. Patients will be randomized to receive treatment with either enzalutamide or flutamide, as shown in Fig. 1.

\section{Participant characteristics Study population}

The study population consists of 100 patients with CRPC who were previously treated with CAB with bicalutamide and whose serum testosterone level is less than $50 \mathrm{ng} / \mathrm{dL}(1.73 \mathrm{nmol} / \mathrm{L})$ and have progressive disease after confirmation of AWS. Disease progression is defined as at least one of the following criteria: PSA progression, soft-tissue disease progression, or bone disease progression according to the Prostate Cancer Working Group 2 criteria [17].

\section{Eligibility criteria}

The inclusion criteria are as follows: 


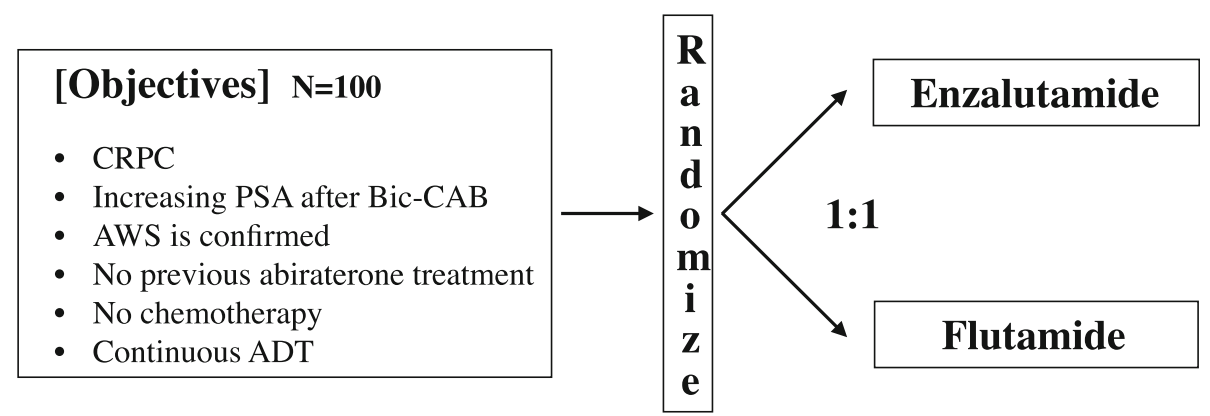

\section{[Primary endpoint]}

- PSA response rate at 3 months

[Secondary endpoint]

- PSA response rate at 6 months

- PSA progression rate at 3/6 months

- PSA progression-free survival

- QOL

- Safety

Fig. 1 The design of the OCUU-CRPC study

1. Serum testosterone of less than $50 \mathrm{ng} / \mathrm{dL}$

2. Disease progression diagnosed on imaging or PSA progression (i.e., consecutive increase of all PSA values measured at least thrice at a 1-week interval and a final value of $2 \mathrm{ng} / \mathrm{mL}$ or more. If the third value is not higher than the second one, a fourth measurement will be taken and its value must be higher than the second one in order for the patient to qualify)

3. Disease progression after $\mathrm{CAB}$ with bicalutamide

4. Eastern Cooperative Oncology Group performance status (PS) of 0 or 1

5. Age 20 years or older

6. Written informed consent

The exclusion criteria are as follows:

1. Any prior treatment with enzalutamide, flutamide, abiraterone, or chemotherapy, except for neoadjuvant therapy

2. Presence of active double cancer

3. Any prior treatment with bicalutamide within 6 weeks

4. Systemic biological therapy (except for existing approved drug as bone-modifying agents or treatment with LHRH analogues) or treatment with other antitumor agents for prostate cancer

5. Presence of severe complications

6. History of hypersensitivity to enzalutamide or any other excipient of enzalutamide
7. History of hypersensitivity to flutamide-containing agent

8. Liver dysfunction

9. Participants who are considered as ineligible by the investigator

\section{Methods of recruitment and random allocation}

Patient recruitment started in January 2015 and is targeted to end by March 2018. Eligible patients are randomly assigned to one of the two treatment groups through the data center at DOT International Inc. (which was responsible for data entry, coding, security, and storage, including any related processes to promote data quality). Patients will be randomly allocated to the enzalutamide or flutamide group via dynamic allocation using metastatic condition (M0, M1) and baseline PSA level as prognostic factors.

\section{Treatment}

Patients were randomly assigned at a 1:1 ratio to the enzalutamide $(160 \mathrm{mg} /$ day, $4 \times 40 \mathrm{mg}$ capsules once daily) or the flutamide ( $375 \mathrm{mg} /$ day; $3 \times 125 \mathrm{mg}$ tablets thrice daily) group according to disease stage (M0 or M1). M0 means the absence of bone metastases on bone scan and of soft-tissue disease, while M1 means bone metastases on bone scan or soft-tissue metastases including nodal involvement above the aortic bifurcation. Both treatments are performed within the coverage of the National Health Insurance privilege of the patients. If the patients satisfy the criteria defined below for 
changing the drug, the initial medication used will be discontinued. The subsequent medication is not specified, and the investigator will choose the appropriate treatment option based on the patient condition. Enzalutamide is expected to be the primary drug for the subsequent treatment of patients in the flutamide group.

\section{Criteria for changing the drug}

The treatment will be changed in case of the following conditions:

1. Disease progression, as defined below (either of one)

a) PSA progression: Three consecutive increases in PSA, an increase of $\geq 25 \%$, and an absolute increase of $\geq 2 \mathrm{ng} / \mathrm{mL}$ above baseline

b) Disease progression as noted on radiography

c) Worsening of symptoms

2. Adverse effects that cause difficulty in continuing the medication

\section{Discontinuation of the treatment}

The investigator will discontinue the treatment or the study and record the reasons for such when the following conditions occur:

1. Patient withdraws from the study

2. Patient died

3. Patient cannot continue the treatment because of hospital transfer

4. The patient was found to be ineligible for the study

5. Any other cases where the investigator determines that the treatment can be discontinued

\section{Endpoints of the study}

The primary endpoint of the study is a PSA response rate (i.e., the ratio of patients whose PSA decreased by $\geq 50 \%$ from baseline) at 3 months. Meanwhile, the secondary endpoints in the OCUU-CRPC study are as follows:

1. PSA progression rate at 3 months

2. PSA response rate at 6 months: If initial enzalutamide therapy is switched to other treatments due to disease progression before 6 months, such cases are regarded as "nonresponders" regardless of the efficacy of the subsequent treatment. In addition, the PSA response rate in patients in whom flutamide is switched to enzalutamide will be calculated to determine the efficacy of enzalutamide in the flutamide to enzalutamide cohort.

3. PSA progression rate at 6 months

4. Change in quality of life (QOL) as assessed using the Functional Assessment of Cancer TherapyProstate (FACT-P) scale in Japanese

5. PSA progression-free survival that is calculated for the initial drug in each arm

6. Adverse events (AEs)

\section{Items to record and schedule}

1. Patient information during initial diagnosis of prostate cancer including the following:date of birth, diagnosis date, age, PSA level, Gleason score, clinical stage, metastatic sites, and rate of bone metastases

2. Patient information during registration (Table. 1):

Table 1 Follow-up schedule of examination

\begin{tabular}{|c|c|c|c|c|c|c|c|}
\hline & Baseline & 1 month & 2 months & 3 months & Every 1 month & Every 3 months & $\begin{array}{l}\text { Discontinuation } \\
\text { of therapy }\end{array}$ \\
\hline Informed consent & $\circ$ & & & & & & \\
\hline Medical history & $\circ$ & & & & & & \\
\hline Confirmation of eligibility criteria & $\circ$ & & & & & & \\
\hline Randomization & $\circ$ & & & & & & \\
\hline Serum testosterone & $\circ$ & & & & & & \\
\hline FACT-P & $\circ$ & - & - & $\circ$ & - & $\circ$ & $\circ$ \\
\hline$P S A^{a}$ & $\circ$ & $\circ$ & $\circ$ & $\circ$ & $\circ$ & $\circ$ & $\circ$ \\
\hline Biochemical examination $^{a}$ & $\circ$ & $\circ$ & $\circ$ & $\circ$ & $\circ$ & $\circ$ & $\circ$ \\
\hline$C T$, bone scintigraphy & $\circ$ & \multicolumn{5}{|c|}{ When progressive disease is suspected ${ }^{b}$} & $\circ$ \\
\hline Safety & $\circ$ & $\circ$ & $\circ$ & $\circ$ & $\circ$ & $\circ$ & $\circ$ \\
\hline Further therapy & - & - & - & \multicolumn{4}{|c|}{ Best standard care } \\
\hline
\end{tabular}

${ }^{a}$ After the discontinuation of enzalutamide or flutamide, PSA and biochemical examination will be continued until September 2018

${ }^{b}$ When progressive disease is suspected, $C T$ and bone scintigraphy will be performed to confirm radiographic disease progression 
a. Patient background data: institution ID, registration date, date of birth, age, height, body weight, PS, concomitant disease, blood sampling (PSA, serum testosterone, white blood cell (WBC), red blood cell (RBC), alkaline phosphatase (ALP), and lactate dehydrogenase (LDH), among others)

b. Imaging: computed tomography $(\mathrm{CT})$ and bone scintigraphy shall be performed on registration to verify metastatic sites

c. QOL (FACT-P)

3. Follow-up after registration (Table. 1);

a. Blood sampling (PSA, WBC, RBC, ALP, and LDH, among others): every month

b. AEs as classified according to the CTCAE ver. 4.0: every month

c. Imaging test: $\mathrm{CT}$ and bone scintigraphy when progressive disease is suspected

d. QOL (FACT-P): every 3 months

\section{Study period}

Registration period: 3 years and 3 months (January 2015-March 2018).

Follow-up period: 6 months after the last patient registration.

\section{Ethical consideration and study registration}

The study will be performed in accordance with the Declaration of Helsinki and will comply to the International Conference on Harmonization and Good Clinical Practice. All possible treatments and examinations for CRPC are undertaken after obtaining written informed consent from the patients before registration. The OCUU-CRPC study received approval from the institutional ethics committees of the participating institutions. The study has been registered at clinicaltrials.gov (NCT02346578) and the University Hospital Medical Information Network (UMIN000016301).

\section{Calculation of the target sample size}

We hypothesize that the PSA response rate (PSA reduction by $\geq 50 \%$ ) of enzalutamide at 3 months is $75 \%$. According to the PREVAIL study [16], the best PSA response rate was $78 \%$. Moreover, most patients in that study achieved a $\geq 50 \%$ PSA response at 3 months. Therefore, we can assume $75 \%$ as the PSA response rate at 3 months in the enzalutamide arm. On the other hand, the PSA response rate of flutamide is $35 \%$ [9]. The primary efficacy endpoint is PSA response rate at 3 months after the initial treatment. A total of 41 patients per group will provide an $90 \%$ power to detect an absolute difference in response rate of $50 \%(75 \%$ vs. $25 \%)$ at 2 -sided $\alpha$ of 0.05 for superiority test with $5 \%$ margin. A dropout rate of $10 \%$ is expected; thus, $N=50$ per group is considered to be adequate.

\section{Discussion}

In the PREVAIL study, enzalutamide significantly reduced the risk of radiographic progression and death by 81\% (HR: $0.19 ; P<0.0001$ ) and 29\% (HR 0.71, $P<0.0001$ ), respectively, compared with a placebo in chemo-naive men with metastatic CRPC [16]. In this study, 61 Japanese were enrolled (enzalutamide, $N=28$; placebo, $N=33$ ), and most them received more than two types of anti-androgens prior to enrollment, including bicalutamide and flutamide. Even after bicalutamide and flutamide, 17 patients $(60.7 \%)$ had confirmed PSA responses ( $\geq 50 \%$ reduction from baseline), and enzalutamide reduced the risk of death by $41 \%$ (HR: 0.59 ; $95 \%$ CI: 0.20 1.78) [18]. Japanese patients reported less baseline pain, had less soft-tissue disease, and had lower median PSA at baseline. ADT is more widely used as an initial treatment for early stages of prostate cancer in Japan than in other countries [19]. Before the ART era, the treatment options for CRPC were limited, and AAT with flutamide has been widely used in Japan. Even after enzalutamide and abiraterone were introduced, Bic-CAB as primary therapy and AAT with flutamide as the subsequent therapy for advanced prostate cancer were commonly used in Japan.

In the OCUU-CRPC study, we compare the efficacy and safety of enzalutamide and AAT with flutamide in patients with CRPC who were previously treated with $\mathrm{CAB}$ therapy with bicalutamide. Because enzalutamide was superior to bicalutamide in terms of PSA response in patients with CRPC who did not receive any prior bicalutamide or chemotherapy in the TERRAIN and STRIVE studies [20, 21], the expected result of the primary endpoint of OCUU-CRPC study will be that enzalutamide is superior to flutamide in terms of PSA response. A longer time to disease progression with enzalutamide than bicalutamide or flutamide may translate to better overall survival, and enzalutamide may be more effective for men with low-volume disease according to post hoc analysis of the PREVAIL trial [22]. However, AAT with flutamide was particularly effective for patients with CRPC who had long response duration to Bic-CAB [8], and the effect of enzalutamide after AAT with flutamide was preserved [18]. Because enzalutamide and flutamide costs $\$ 2478$ and $\$ 208$ ( $\$ 118$ if generic drug) per month in Japan, respectively, AAT with flutamide may be more beneficial and accessible for limited patients owing to its lower cost.

The OCUU-CRPC study has some limitations. The PSA response rate at 3 months without radiographic examinations as the primary endpoint may not be associated directly with overall survival. Moreover, this is not a crossover trial and the treatment after enzalutamide 
and flutamide is not defined by the protocol. Additionally, because the patients included in this study are Japanese and because flutamide is not commonly used for CRPC in other countries, the findings of the OCUU-CRPC study cannot be generalized and applied to patient populations in other locations or regions.

Currently, a similar clinical trial with enzalutamide and flutamide for CRPC (NCT02918968) is undergoing in Japan, in which the primary endpoint is time to PSA progression with first-line therapy. The result of the NCT02918968 will be published a few years after our result.

\section{Abbreviations}

AAT: Alternative antiandrogen therapy; ADT: Androgen-deprivation therapy; AEs: Adverse events; ALP: Alkaline phosphatase; ART: Androgen receptor targeted therapy; AWS: Antiandrogen withdrawal syndrome; CAB Bic: Combined androgen blockade therapy with bicalutamide; CAB: Combined androgen blockade; CRPC: Castration-resistant prostate cancer; CT: Computed tomography; CTCAE: Common Terminology Criteria for Adverse Events; ECOG-PS: Eastern Cooperative Oncology Group performance status; FACT-P: The Functional Assessment of Cancer TherapyProstate; LDH: Lactate dehydrogenase; LHRH: Luteinizing hormone-releasing hormone; PSA: Prostate specific antigen; QOL: Quality of life; RBC: Red blood cell; WBC: White blood cell

\section{Acknowledgements}

The following individuals and institutions participated in OCUU-CRPC study: K. Kuratsukuri, N. Kuwabara, T. Iwai, Osaka City University, Osaka, Japan; T. Ohmachi, Bellland General Hospital, Sakai, Japan; K. Harimoto, C. Masuda, Y. Kuroki, Fuchu Hospital, Izumi, Japan; S. Yamamoto, Ikuwakai Memorial Hospital, Osaka, Japan; Y. Yamakoshi, Ishikiriseiki Hospital, Higashi-osaka, Japan; H. Iwata, K. Uemiya, Itami Municipal Hospital, Itami, Japan; K. Tashiro, Meijibashi Hospital, Matsubara, Japan; Y. Takegaki, Minamiosaka Hospital, Osaka, Japan; M. Yoshimoto, S. Sugita, Ohno Memorial Hospital, Osaka, Japan; T. Sugimoto, W. Sakamoto, K. Ishii, Osaka City General Hospital, Osaka, Japan; T. Adachi, C. Nishihara, Osaka City Juso Hospital, Osaka, Japan; Y. Yoneda, Y. Komemushi, Osaka General Hospital of West Japan Railway Company, Osaka, Japan; T. Ito, T. Sonoda, T. Deguchi, PL Hospital, Tondabayashi, Japan; S. Tanabe, M. Mitsuhashi, Shirasagi Hospital, Osaka, Japan; N. Kumada, J. Matsuda, T. Hase Suita Municipal Hospital, Suita, Japan; S. Ikemoto, M. Kamizuru, Y. Machida, Yao Municipal Hospital, Yao, Japan; H. limori, T. Kanamaru, Yao Tokushukai General Hospital, Yao, Japan.

\section{Funding}

This study was funded by Astellas pharma Inc. However, all other processes including the study design, collection of data, analyses, interpretations, and the writing of the manuscript were the responsibilities of Osaka City University.

\section{Availability of data and materials}

The data of this article is not available until the final report of this study to avoid bias toward the analysis.

\section{Authors' contributions}

TI drafted the manuscript and had full access to all the data in the study and had final responsibility for the decision to submit for publication. TI, ST, MK, TY and TN planned, coordinated, and conducted the study. SY contributed as a clinical research coordinator. All authors read and approved the final manuscript. All other participants in this study contributed to the enrollment, treatment, and follow-up of patients.

\section{Ethics approval and consent to participate}

The OCUU-CRPC study was approved by ethical committee in Osaka City University Graduate School of Medicine on September 292,014 (reference number: 2936) while the study protocol was amended on November 282,016 (ver. 5), and subsequently from the institutional ethics committees of all other participating 16 hospitals listed below; Bellland General Hospital:
Approved on 23 Feb 2015. Fuchu Hospital: Approved on 17 Dec 2014. Ikuwakai Memorial Hospital: Approved on 3 Feb 2015. Ishikiriseiki Hospital: Approved on 9 Jan 2015. Itami Municipal Hospital: Approved on 18 May 2015. Meijibashi Hospital: Approved on 8 Jun 2015. Minamiosaka Hospital: Approved on 19 Dec 2014. Ohno Memorial Hospital: Approved on 26 Feb 2015. Osaka City General Hospital: Approved on 11 May 2015. Osaka City Juso Hospital: Approved on 30 Jan 2015. Osaka General Hospital of West Japan Railway Company: Approved on 13 Jan 2016. PL Hospital: Approved on 7 Sep 2015. Shirasagi Hospital: Approved on 24 Feb 2015. Suita Municipal Hospital: Approved on 7 Aug 2015. Yao Municipal Hospital: Approved on 16 Jan 2015. Yao Tokushukai General Hospital: Approved on 22 Jun 2015.

\section{Consent for publication}

Not applicable.

\section{Competing interests}

T. Iguchi and T. Nakatani have research funding from Astellas pharma Inc. and Bayer Yakuhin, Ltd. The other authors declare that they have no competing interests.

\section{Publisher's Note}

Springer Nature remains neutral with regard to jurisdictional claims in published maps and institutional affiliations.

Received: 21 November 2017 Accepted: 27 March 2019

Published online: 11 April 2019

\section{References}

1. Siegel RL, Miller KD, Jemal A. Cancer statistics, 2017. CA Cancer J Clin. 2017; 67(1):7-30.

2. Akaza H, Homma Y, Usami M, Hirao Y, Tsushima T, Okada K, Yokoyama M, Ohashi Y, Aso Y, Prostate Cancer Study G. Efficacy of primary hormone therapy for localized or locally advanced prostate cancer: results of a 10year follow-up. BJU Int. 2006;98(3):573-9.

3. Akaza H, Hinotsu S, Usami M, Arai Y, Kanetake H, Naito S, Hirao Y, Study Group for the Combined Androgen Blockade Therapy of Prostate C. Combined androgen blockade with bicalutamide for advanced prostate cancer: long-term follow-up of a phase 3, double-blind, randomized study for survival. Cancer. 2009;115(15):3437-45.

4. Ueno S, Namiki M, Fukagai T, Ehara H, Usami M, Akaza H. Efficacy of primary hormonal therapy for patients with localized and locally advanced prostate cancer: a retrospective multicenter study. Int J Urol. 2006;13(12):1494-500.

5. Fujikawa K, Matsui $Y$, Fukuzawa S, Takeuchi H. Prostate-specific antigen levels and clinical response to flutamide as the second hormone therapy for hormone-refractory prostate carcinoma. Eur Urol. 2000;37(2):218-22.

6. Miyake $H$, Hara I, Eto $H$. Clinical outcome of maximum androgen blockade using flutamide as second-line hormonal therapy for hormone-refractory prostate cancer. BJU Int. 2005;96(6):791-5.

7. Okihara K, Ukimura O, Kanemitsu N, Mizutani Y, Kawauchi A, Miki T. Clinical efficacy of alternative antiandrogen therapy in Japanese men with relapsed prostate cancer after first-line hormonal therapy. Int J Urol. 2007;14(2):128-32.

8. Nishimura K, Arichi N, Tokugawa S, Yoshioka I, Kishikawa H, Ichikawa Y. Effects of flutamide as a second-line agent for maximum androgen blockade of hormone refractory prostate cancer. Int J Urol. 2007;14(3):264-7.

9. Suzuki H, Okihara K, Miyake H, Fujisawa M, Miyoshi S, Matsumoto T, Fujii M, Takihana Y, Usui T, Matsuda T, et al. Alternative nonsteroidal antiandrogen therapy for advanced prostate cancer that relapsed after initial maximum androgen blockade. J Urol. 2008;180(3):921-7.

10. Okegawa T, Nutahara K, Higashihara E. Alternative antiandrogen therapy in patients with castration-resistant prostate cancer: a single-center experience. Int J Urol. 2010;17(11):950-5.

11. Yasui M, Uemura K, Yoneyama S, Kawahara T, Hattori $Y$, Teranishi Jl, Inoue M, Ohta Jl, Yokomizo Y, Yao M, et al. Predictors of poor response to secondary alternative antiandrogen therapy with flutamide in metastatic castration-resistant prostate cancer. Jpn J Clin Oncol. 2016;46(11):1042-6.

12. Lowrance WT, Roth BJ, Kirkby E, Murad MH, Cookson MS. Castrationresistant Prostate Cancer: AUA guideline amendment 2015. J Urol. 2016; 195(5):1444-52.

13. Tran C, Ouk S, Clegg NJ, Chen Y, Watson PA, Arora V, Wongvipat J, SmithJones PM, Yoo D, Kwon A, et al. Development of a second-generation 
antiandrogen for treatment of advanced prostate cancer. Science (New York, NY). 2009;324(5928):787-90

14. Jung ME, Ouk S, Yoo D, Sawyers CL, Chen C, Tran C, Wongvipat J. Structureactivity relationship for thiohydantoin androgen receptor antagonists for castration-resistant prostate cancer (CRPC). J Med Chem. 2010;53(7):2779-96.

15. Scher HI, Fizazi K, Saad F, Taplin ME, Sternberg CN, Miller K, de Wit R, Mulders P, Chi KN, Shore ND, et al. Increased survival with enzalutamide in prostate cancer after chemotherapy. N Engl J Med. 2012;367(13):1187-97.

16. Beer TM, Armstrong AJ, Rathkopf DE, Loriot $Y$, Sternberg CN, Higano CS, Iversen P, Bhattacharya S, Carles J, Chowdhury S, et al. Enzalutamide in metastatic prostate cancer before chemotherapy. N Engl J Med. 2014;371(5):424-33.

17. Scher HI, Halabi S, Tannock I, Morris M, Sternberg CN, Carducci MA, Eisenberger MA, Higano C, Bubley GJ, Dreicer R, et al. Design and end points of clinical trials for patients with progressive prostate cancer and castrate levels of testosterone: recommendations of the Prostate Cancer clinical trials working group. J Clin Oncol. 2008;26(7):1148-59.

18. Kimura G, Yonese J, Fukagai T, Kamba T, Nishimura K, Nozawa M, Mansbach $H$, Theeuwes A, Beer TM, Tombal B, et al. Enzalutamide in Japanese patients with chemotherapy-naive, metastatic castration-resistant prostate cancer: a post-hoc analysis of the placebo-controlled PREVAIL trial. Int J Urol. 2016; 23(5):395-403

19. Namiki M, Akaza H, Lee SE, Song JM, Umbas R, Zhou L, Lee BC, Cheng C, Chung MK, Fukagai T, et al. Prostate Cancer working group report. Jpn J Clin Oncol. 2010;40(Suppl 1):170-5.

20. Shore ND, Chowdhury S, Villers A, Klotz L, Siemens DR, Phung v OS, Hasabou N, Wang F, Bhattacharya S, et al. Efficacy and safety of enzalutamide versus bicalutamide for patients with metastatic prostate cancer (TERRAIN): a randomised, double-blind, phase 2 study. Lancet Oncol. 2016;17(2):153-63.

21. Penson DF, Armstrong AJ, Concepcion R, Agarwal N, Olsson C, Karsh L, Dunshee C, Wang F, Wu K, Krivoshik A, et al. Enzalutamide versus Bicalutamide in castration-resistant Prostate Cancer: the STRIVE trial. J Clin Oncol. 2016;34(18):2098-106.

22. Evans CP, Higano CS, Keane T, Andriole G, Saad F, Iversen P, Miller K, Kim CS, Kimura G, Armstrong AJ, et al. The PREVAlL Study: primary outcomes by site and extent of baseline disease for enzalutamide-treated men with chemotherapy-naive metastatic castration-resistant Prostate Cancer. Eur Urol. 2016;70(4):675-83.

Ready to submit your research? Choose BMC and benefit from:

- fast, convenient online submission

- thorough peer review by experienced researchers in your field

- rapid publication on acceptance

- support for research data, including large and complex data types

- gold Open Access which fosters wider collaboration and increased citations

- maximum visibility for your research: over $100 \mathrm{M}$ website views per year

At $\mathrm{BMC}$, research is always in progress.

Learn more biomedcentral.com/submissions 